\title{
Microflora subgingival en periodontitis crónica y agresiva en Bogotá, Colombia: un acercamiento epidemiológico
}

\author{
Isabel Mayorga-Fayad ${ }^{~}$, Gloria I. Lafaurie ${ }^{1}$, Adolfo Contreras ${ }^{2}$, Diana M. Castillo ${ }^{1}$, \\ Alexandra Barón ${ }^{1}$, María del Rosario Aya \\ 1 Laboratorio de Microbiología Oral, Instituto UIBO (Unidad de Investigación Básica Oral), Universidad \\ El Bosque, Bogotá, D.C., Colombia. \\ 2 Grupo de Medicina Periodontal, Universidad del Valle, Cali, Colombia.
}

Introducción. Los microorganismos involucrados en la periodontitis varían en cada región dependiendo de diferentes factores. Por ello cada país debe establecer su propio perfil microbiológico con el objeto de proponer esquemas adecuados de prevención y tratamiento. Objetivo. Investigar la presencia y concentración subgingival de Porphyromonas gingivalis, Tannerella forsythia, Actinobacillus actinomycetemcomitans, Prevotella intermedia/Prevotella nigrescens, Eikenella corrodens, Campylobacter rectus, Micromonas micros, Fusobacterium spp, Dialister pneumosintes y bacilos entéricos en pacientes con periodontitis en una población colombiana considerando edad, género y nivel socioeconómico.

Materiales y métodos. Participaron 183 pacientes: 84 con periodontitis crónica, 59 con periodontitis agresiva y 40 individuos sanos. De cada paciente se obtuvo un grupo de muestras de los seis sitios con mayor profundidad de bolsa que se procesaron por cultivo para el aislamiento e identificación de las bacterias.

Resultados. En periodontitis crónica y agresiva se detectaron con alta y similar frecuencia $P$. gingivalis, $T$. forsythia, $P$. intermedia/P. nigrescens, $C$. rectus, Fusobacterium spp y E. corrodens y con menor y similar frecuencia, M. micros, A. actinomycetemcomitans, D. pneumosintes y bacilos entéricos, principalmente de la tribu Klebsielleae. En los sujetos sanos se encontraron con frecuencia elevada $P$. intermedia/ $P$. nigrescens, $C$. rectus y Fusobacterium spp y con frecuencia moderada, $M$. micros y E. corrodens.

Conclusión. Los resultados indican que $P$. gingivalis, $T$. forsythia, $P$. intermedia/P. nigrescens, C. rectus, Fusobacterium spp y E. corrodens constituyen parte importante del perfil microbiológico de las periodontitis en la población colombiana. En la periodontitis agresiva, $P$. gingivalis se encontró más frecuentemente que $A$. actinomycetemcomitans.

Palabras clave: periodontitis/epidemiología, Porphyromonas gingivalis, bacterias anaerobias, Enterobacteriaceae, Pseudomonadaceae.

Subgingival microbiota in chronic and aggressive periodontitis in Bogotá, Colombia: an epidemiological approach

Introduction. Periodontitis-associated microbiotas differ in regions around the world and vary with a variety of factors. This suggests that each country must establish its own dental microbiobiotic profile in order to formulate adequate prevention measures and more specific treatments.

Objective. The prevalence and proportions of the following microorganisms were investigated: Porphyromonas gingivalis, Tannerella forsythia, Actinobacillus actinomycetemcomitans, Prevotella intermedia/Prevotella nigrescens, Eikenella corrodens, Campylobacter rectus, Micromonas micros, Fusobacterium spp, Dialister pneumosintes and enteric rods. The patients selected had been diagnosed with chronic periodontitis and aggressive periodontitis from clinical populations in Bogotá, Colombia. The variables of age, gender and socioeconomic level were included in the comparisons.

Materials and methods. 183 patients were classified in two groups, chronic periodontitis $(n=84)$ and aggressive periodontitis $(n=59) ; 40$ healthy subjects were selected for comparison. $A$ 
pooled subgingival plaque sample was obtained from the 6 deepest periodontal pockets in every patient and processed by culture for the isolation and identification of bacteria.

Results. In chronic periodontitis and aggressive periodontitis, $P$. gingivalis, $T$. forsythia, $P$. intermedia/P. nigrescens, $C$. rectus, Fusobacterium spp and $E$. corrodens showed high and similar prevalence, whereas $M$. micros, $A$. actinomycetemcomitans, $D$. pneumosintes and enteric rods (commonly from the tribe Klebsielleae) showed less and similar prevalence. In the healthy group, $P$. intermedia/P. nigrescens, $C$. rectus and Fusobacterium spp showed high frequency whereas $M$. micros and $E$. corrodens showed moderate frequency.

Conclusions. Porphyromonas gingivalis, T. forsythia, $P$. intermedia/P. nigrescens, C. rectus, Fusobacterium spp. and E. corrodens are important constituents of the microbiologic profile of the periodontitis in Colombian populations. Porphyromonas gingivalis was found more frequently than $A$. actinomycetemcomitans in aggressive periodontitis.

Key words: periodontitis/epidemiology, Porphyromonas gingivalis, anaerobic bacteria, Enterobacteriaceae, Pseudomonadaceae.

La periodontitis es una enfermedad infecciosa que genera inflamación de los tejidos de soporte dental, pérdida de inserción progresiva y pérdida ósea, caracterizada por la formación de bolsas que pueden afectar un número variable de dientes y que muestra diferentes velocidades de progresión. El Consenso de la Academia Americana de Periodoncia (AAP, 1999) estableció las diferencias entre las formas crónica y agresiva de la periodontitis basándose en los parámetros clínicos, la composición microbiológica de la placa dental subgingival y los aspectos inmunológicos del huésped (1).

Diversos datos epidemiológicos indican que la periodontitis se presenta con alta prevalencia en la población mundial (2-7). En Colombia, la enfermedad periodontal evaluada mediante la pérdida de inserción clínica afecta al $50 \%$ de la población. En su forma generalizada, el $12 \%$ de los individuos menores de 35 años muestra pérdida de inserción, la que aumenta a $42 \%$ después de los 60 años. En la forma avanzada, el 10\% de la población presenta pérdida de inserción avanzada (8).

La placa bacteriana es una estructura no calcificada constituida por componentes salivares y numerosos géneros bacterianos en crecimiento continuo. El término "biopelícula" se usa para

\footnotetext{
Correspondencia:

Isabel Mayorga de Fayad, transversal 9A Bis No. 132-55, Bogotá, D.C, Colombia.

Teléfono: (571) 6331368 ext 158 y 345, fax: (571) 625 2030

mayorgaisabel@unbosque.edu.co

Recibido: 12/05/06; aceptado: 30/10/06
}

denotar una comunidad de bacterias envueltas en una masa extracelular polimérica que se acumula en una superficie. Las diferentes especies acumuladas en dicha estructura pueden proteger contra la colonización por patógenos exógenos o constituir el inicio, progresión y destrucción de las estructuras periodontales $(9,10)$. Algunas especies como Porphyromonas gingivalis, Tannerella forsythia, Actinobacillus actinomycetemcomitans, Prevotella intermedia/Prevotella nigrescens, Eikenella corrodens, Campylobacter rectus y Treponema denticola aumentan en frecuencia y concentración en la biopelícula de individuos con periodontitis en comparación con la población sana. La presencia de altas concentraciones de $P$. gingivalis, $T$. forsythia y $A$. actinomycetemcomitans se asocian con la progresión de las lesiones periodontales en pacientes tratados y no tratados $(4,11)$.

La distribución de los microorganismos en la placa dental subgingival varía de un país a otro dependiendo del área geográfica, raza, dieta, nivel de desarrollo y condiciones de vida, entre otros $(12,13)$, por lo que se recomienda que cada país debe establecer su propio perfil microbiológico en los pacientes con periodontitis. Los microorganismos más frecuentemente aislados de la bolsa periodontal han sido muy estudiados en Norte América $(7,11,14-19)$ y Europa (20-23). Sin embargo, el perfil microbiológico de la periodontitis en poblaciones de Asia (24-27), África (12,28-30), Centro (31) y Sur América (32-37) parece diferir en frecuencia y composición debido, en parte, al frecuente hallazgo de microflora no usual. Por 
ejemplo, los bacilos entéricos de las familias Enterobacteriaceae y Pseudomonadaceae producen factores de virulencia que podrían afectar el comportamiento clínico de la periodontitis, así como su control y tratamiento. En Centro y Sur América, el componente microbiológico de la periodontitis tanto crónica como agresiva solamente se ha analizado en detalle en algunos países como República Dominicana (31), Brasil (32-34), Chile (35-37) y Argentina (38). El propósito del presente estudio fue investigar la presencia y concentración de $P$. gingivalis, $T$. forsythia, $A$. actinomycetemcomitans, $P$. intermedia/P. nigrescens, E. corrodens, C. rectus, Micromonas micros, Fusobacteriumspp, Dialister pneumosintes y bacilos entéricos (familias Enterobacteriaceae y Pseudomonadaceae) en pacientes con periodontitis crónica y agresiva en una población de Bogotá, Colombia, para lo cual se evaluaron variables como edad, género y nivel socioeconómico.

\section{Materiales y métodos \\ Población}

Participaron en este estudio 183 pacientes: 84 con diagnóstico de periodontitis crónica, 59 con diagnóstico de periodontitis agresiva y un grupo control de 40 sujetos. Todos los pacientes eran residentes de la ciudad de Bogotá D.C., Colombia, y fueron remitidos de la práctica privada y de servicios públicos institucionales en convenio con la Universidad El Bosque. Otros asistían regularmente a la Clínica de Postgrado de Periodoncia y Medicina Oral de esta institución. A todos los pacientes se les realizó un examen periodontal que incluyó profundidad de bolsa (se utilizó una sonda periodontal UNC-15. Hu Friedy, Chicago, IL, USA), nivel de inserción, sangrado y supuración y fueron clasificados de acuerdo con las recomendaciones del consenso de la Academia Americana de Periodoncia de 1999 (1).

En el grupo con periodontitis crónica se aceptaron pacientes con periodontitis crónica generalizada y periodontitis crónica localizada con por lo menos cuatro sitios con bolsas mayores o iguales a 5 $\mathrm{mm}$. Para periodontitis agresiva se aceptaron periodontitis agresiva generalizada y periodontitis agresiva localizada con por lo menos seis sitios con bolsas mayores o iguales a $7 \mathrm{~mm}$, teniendo en cuenta una pérdida generalizada y avanzada que comprometiera incisivos y primeros molares (para periodontitis agresiva localizada), e incisivos, primeros molares y tres dientes adicionales (para periodontitis agresiva generalizada). En el grupo control se aceptaron pacientes sin periodontitis de 20 años de edad o mayores, mínimo 24 dientes, ausencia de bolsas periodontales y de pérdida de inserción en áreas proximales, menos de $30 \%$ de sitios con encías rojas o hemorragia al sondaje y buena salud general establecida por anamnesis.

Fueron excluidos los pacientes diabéticos y con enfermedades sistémicas, pacientes que hubieran tenido terapia periodontal en el último año y que hubieran consumido antibióticos, corticoides o analgésicos no esteroideos en los seis meses anteriores a la toma de la muestra. A los pacientes con diagnóstico de periodontitis agresiva se les realizaron exámenes de glicemia y cuadro hemático para evaluar el recuento de polimorfonucleares neutrófilos y descartar compromisos sistémicos asociados con la periodontitis.

Para la calibración de los investigadores clínicos en la selección de los pacientes y la toma de las muestras se diseñó un disco compacto interactivo con 10 casos clínicos totalmente documentados con anamnesis, examen clínico periodontal, fotografía clínica, radiografías, exámenes complementarios y una guía de criterios de selección basados en las recomendaciones diagnósticas del Consenso Internacional de la Academia Americana de Periodoncia de 1999 (1).

Antes de la iniciación del estudio se llevó a cabo un taller de calibración diagnóstica en la sede de la Universidad El Bosque, cuya metodología se basó en la presentación de casos y la discusión tanto del diagnóstico como de la elegibilidad para el estudio, y se unificaron criterios por consenso. Se realizaron ejercicios de calibración para los parámetros clínicos hasta lograr un nivel entre examinadores del $90 \%$ para profundidad de la bolsa y del $80 \%$ para nivel de inserción clínico.

Además, a los pacientes con diagnóstico de periodontitis se les brindó un entrenamiento en la utilización y toma de índices clínicos, manejo de formatos para envío al laboratorio y toma de muestras de placa subgingival. 
Este estudio fue aceptado por el Comité Institucional de Ética de la Universidad El Bosque y todos los pacientes aceptaron voluntariamente su participación y firmaron un consentimiento informado.

Se recolectaron los siguientes datos para cada paciente: edad (años cumplidos), género (femenino/masculino) y nivel socioeconómico (bajo: estratos 1 y 2 , medio: estratos 3 y 4 y alto: estratos 5 y 6 ) basados en la estratificación del Departamento Nacional de Planeación adoptada por las empresas de servicios públicos para todos los departamentos de Colombia (39).

\section{Toma de la muestra}

Antes de la toma de la muestra se eliminó la placa supragingival con curetas y se aisló el sector con rollos de algodón. A cada paciente se le tomaron seis muestras de los seis sitios con mayor profundidad de bolsa ( $\geq 5 \mathrm{~mm}$ ), para lo cual se insertó una punta de papel absorbente estéril en cada sitio durante 20 segundos. Todas las puntas se recolectaron en un frasco con $2,0 \mathrm{~mL}$ de medio de transporte VMGA III (Viability Medium Göteborg Anaerobically preparado y esterilizado, que mantiene la viabilidad de los microorganismos sin permitir su multiplicación) con el objeto de obtener un grupo para el análisis. Las muestras se llevaron al Laboratorio de Microbiología Oral del Instituto UIBO (Unidad de Investigación Básica Oral) de la Universidad El Bosque en un tiempo no mayor a 24 horas después de tomada la muestra para evitar la pérdida de microorganismos anaerobios o facultativos.

\section{Aislamiento e identificación de microorganismos}

El cultivo e identificación de las especies microbianas se realizó de acuerdo con las recomendaciones de Slots $(40,41)$, cuyas técnicas fueron estandarizadas previamente en nuestro laboratorio. Brevemente, para la identificación de $P$. gingivalis, $T$. forsythia, $P$. intermedia/P. nigrescens, E. corrodens, C. rectus, M. micros, Fusobacterium spp y $D$. pneumosintes, se hicieron cinco diluciones en base 10 a partir del medio VMGA III, se sembraron $100 \mu \mathrm{L}$ de las diluciones $10^{-3}, 10^{-4}$ y $10^{-5}$ en agar brucella sangre enriquecido con hemina y menadiona (BBL Microbiology Systems, Cockeysville, MD) y se llevaron a incubación a $36^{\circ} \mathrm{C}$ en atmósfera de anaerobiosis (Anaerogen, Oxoid, Hampshire, England) durante siete días. Para la identificación de A. actinomycetemcomitans, las muestras sin diluir y $10^{-1}$ se sembraron en agar TSBV (tripticasa soya bacitracina vancomicina) y se incubaron en atmósfera de $10 \%$ de $\mathrm{CO}_{2}$ (Campygen, Oxoid, Hampshire, England) durante tres a cinco días. Para el aislamiento de las bacterias entéricas, la muestra sin diluir se sembró en agar MacConkey, el cual se incubó en aerobiosis durante 24 a 48 horas a $37^{\circ} \mathrm{C}$.

Las colonias de $P$. gingivalis (figura $1 \mathrm{~A}$ ) se identificaron por la morfología y pigmento de la colonia, la prueba de luz ultravioleta negativa y la prueba de CAAM (NCBZ-GLY-GLY-ARGclorohidrato de 7-amido-4-metil coumarina, para la detección de una enzima tipo tripsina) positiva (42). Para T. forsythia (figura 1B), además de las características de la colonia, se utilizó la prueba de CAAM (positiva). C. rectus se reconoció por su movilidad (16) y E. corrodens (figura 1C) con las pruebas de nitritos y oxidasa positivas (19). Se realizaron además pruebas bioquímicas y enzimáticas confirmatorias para todos los microorganismos utilizando los sistemas de identificación comercial Rapid ANA II (Remel ${ }^{\mathrm{TM}}$, Apogent) y API ZYM (Biomerieux® Francia). Las cepas presuntivas de $A$. actinomycetemcomitans (figura 1D) fueron identificadas por la presencia de una estructura semejante a una estrella en el interior de las colonias y las pruebas catalasa positiva y MUG negativa (4-Metilumbeliferil-B-Dgalactosido, para investigar la fermentación de la lactosa) (40).

Las bacterias entéricas se reconocieron por sus características morfológicas y tintoriales y su respuesta a la fermentación de la lactosa. Se utilizó además el sistema de identificación Crystal Enteric/Nonfermenter (BBL®)

\section{Cuantificación de las bacterias}

Después de la incubación se calculó el número total de unidades formadoras de colonias por mililitro de cada muestra a partir de los medios de 


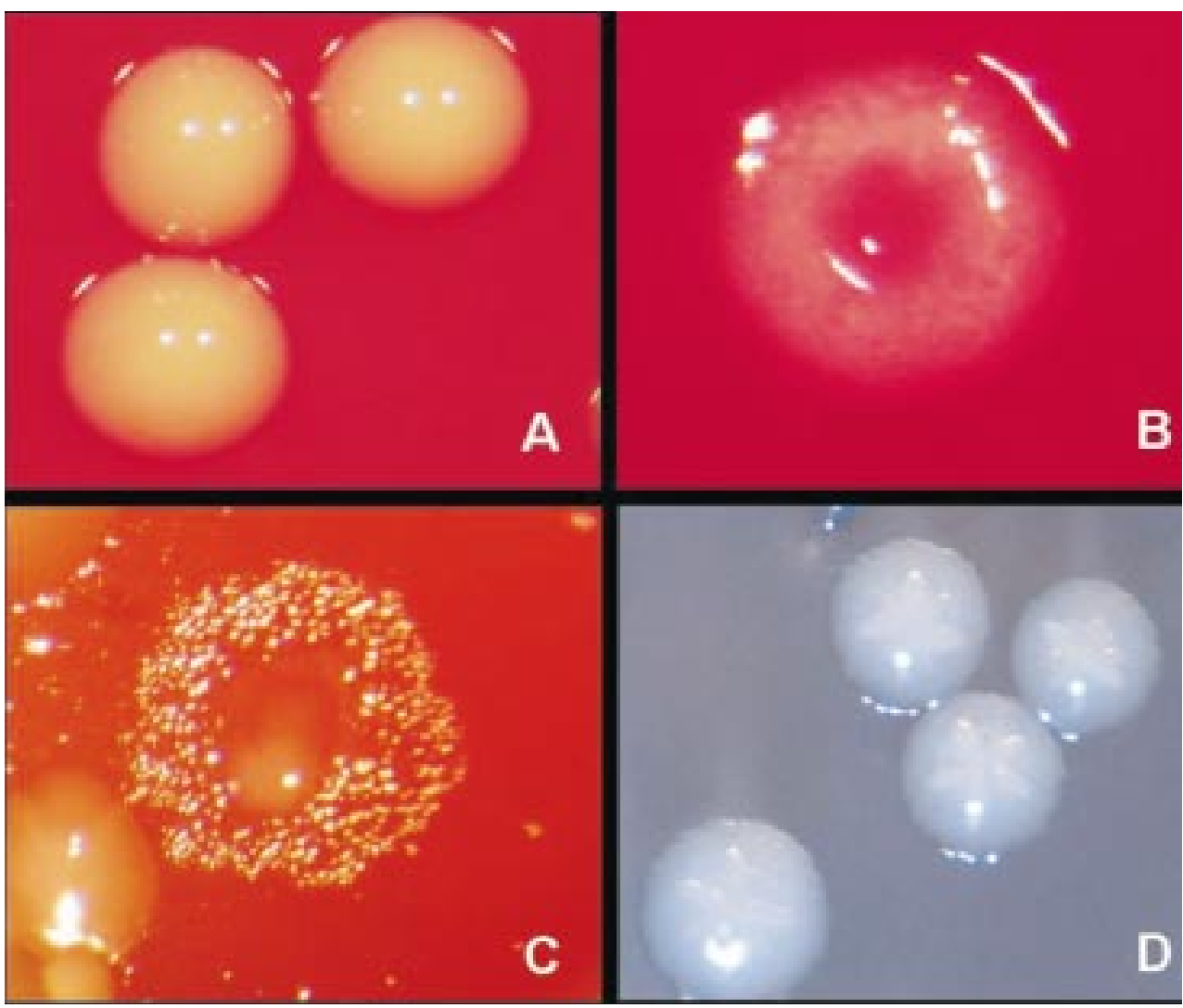

Figura 1. Morfología de las colonias de microorganismos periodontopatógenos observadas con microscopio estereoscópico a 4,5 aumentos (fotografías tomadas en el Laboratorio de Microbiología Oral, Instituto UIBO, Universidad El Bosque). A: P. gingivalis, B: T. forsythia. C: E. corrodens, D: A. actinomycetemcomitans.

cultivo. El porcentaje relativo de recuperación de cada microorganismo se calculó a partir del total de unidades formadoras de colonias por mililitro.

\section{Análisis estadístico}

Se realizó inicialmente un análisis estadístico descriptivo univariado (distribución de frecuencias de variables categóricas, media, mediana y desviación estándar de variables continuas). Posteriormente, se realizó un análisis bivariado entre los perfiles bacterianos (variables dicotómicas) y variables sociodemográficas: edad, género y nivel socioeconómico, mediante pruebas de ji cuadrado y prueba exacta de Fisher cuando el tamaño de casos en las celdas de análisis era menor a cinco. Se hicieron comparaciones de valores medios para perfiles bacterianos entre los niveles diagnósticos (crónicos comparados con sanos y agresivos comparados con sanos) utilizando pruebas no paramétricas (prueba de Mann-Whitney). El valor de significación para todas las pruebas se estableció como $p=0,05$.

\section{Resultados}

En el cuadro 1 se presentan los datos clínicos y demográficos de la muestra estudiada. En relación con el género de los sujetos investigados no se encontró diferencia significativa en ninguno de los dos grupos con periodontitis; el grupo control presentó más mujeres que hombres. La edad fue similar en los pacientes con periodontitis agresiva y los controles, aunque la media de edad en los pacientes con periodontitis crónica fue mayor. Fue evidente el mayor avance de los índices clínicos entre los grupos con periodontitis y el grupo control y se observó mayor pérdida de inserción, índice 
de sangrado y profundidad de bolsa en los pacientes con periodontitis agresiva.

En el cuadro 2 se observa el componente microbiano en los tres grupos estudiados designados así: a) periodontitis crónica, b) periodontitis agresiva, c) grupo control. $P$. gingivalis y $T$. forsythia se encontraron en proporción similar tanto en periodontitis crónica $(60,7 \%$ y $73,8 \%$, respectivamente) como en agresiva $(67,8 \%$ y $64,4 \%$, respectivamente). Estos dos grupos de bacterias presentaron diferencias significativas al compararlos con el grupo control $(p<0,001)$, en el cual se encontró $7,5 \%$ de $P$. gingivalis y $10 \%$ de $T$. forsythia.
C. rectus no presentó diferencias significativas en ninguno de los tres grupos, ya que se encontró en elevada proporción tanto en los grupos enfermos como en los sanos. Fusobacterium spp y M. micros se presentaron con mayor frecuencia en los pacientes con periodontitis crónica $(p<0,05$ comparado con el grupo control), pero no se apreciaron diferencias significativas ni entre los dos grupos con periodontitis ni entre los pacientes con periodontitis agresiva y el grupo control. $P$. intermedia/ $P$. nigrescens se presentaron con mayor frecuencia en los dos grupos con periodontitis ( $p<0,05$ comparado con el grupo control), pero no se encontraron diferencias

Cuadro 1. Descripción demográfica y clínica de la muestra estudiada.

\begin{tabular}{|c|c|c|c|}
\hline Parámetro & Periodontitis crónica & Periodontitis agresiva & Grupo control \\
\hline Sujetos (n) & 84 & 59 & 40 \\
\hline Edad (media \pm D.E.) & $46,0 \pm 10,5$ * & $33,0 \pm 9,2$ & $32,6 \pm 10,6$ \\
\hline Género (\%) & $\mathrm{F}: 46,4 \mathrm{M}: 53,6^{* *}$ & $\mathrm{~F}: 51 \mathrm{M}: 49$ ** & $\mathrm{F}: 70 \mathrm{M}: 30$ \\
\hline $\begin{array}{l}\text { Sangrado al sondaje } \\
\text { (\% positivo) } \\
\text { PB sitios muestreados }\end{array}$ & 59,2 * & 70,38 * & 15,3 \\
\hline (Media mm \pm D.E.) & $7,5 \pm 1,7^{\star}$ & $7,99 \pm 3,8^{*}$ & $2,0 \pm 0,8$ \\
\hline PB mm (media \pm D.E.) & $3,9 \pm 2,1$ * & $3,9 \pm 2,3$ * & $1,7 \pm 0,7$ \\
\hline NIC mm (media \pm D.E.) & $3,8 \pm 2,9$ * & $4,0 \pm 3,1$ * & $0,9 \pm 0,9$ \\
\hline
\end{tabular}

PB: Profundidad de bolsa

NIC: Nivel de inserción clínico

${ }^{*} p<0,001$. ${ }^{* *} p<0,05$ comparación entre periodontitis crónica vs. grupo control y periodontitis agresiva vs. grupo control (Prueba ji cuadrado/prueba exacta de Fisher).

Cuadro 2. Frecuencia (\%) de detección de microorganismos periodontopatógenos según presentación clínica.

\begin{tabular}{|c|c|c|c|}
\hline Microorganismo & $\begin{array}{l}\text { Periodontitis crónica } \\
\qquad(n=84)\end{array}$ & $\begin{array}{l}\text { Periodontitis agresiva } \\
\qquad(n=59)\end{array}$ & $\begin{array}{l}\text { Grupo control } \\
(n=40)\end{array}$ \\
\hline P. gingivalis & $60,7^{*} a$ & $67,8^{*} \mathrm{~b}$ & 7,5 \\
\hline T.forsythia & $73,8^{\star} a$ & $64,4^{*} \mathrm{~b}$ & 10 \\
\hline A.actinomycetemcomitans & 14,2 & $22,0^{* *} b$ & 5 \\
\hline P.intermedia/nigrescens & $72,6^{* *} a$ & $74,6^{* * b}$ & 45 \\
\hline E.corrodens & $71,4^{\star} \mathrm{a}$ & $67,8 * b$ & 22,5 \\
\hline C. rectus & 70,2 & 61,0 & 57,5 \\
\hline M.micros & $35,7^{\star *} a$ & 33,9 & 17,5 \\
\hline Fusobacterium spp & $82,1^{\star *} a$ & 71,1 & 60 \\
\hline D.pneumosintes & $15,5^{\star *} a$ & $15,2^{* *} b$ & 2,5 \\
\hline Bacilos entéricos & 13,1 & 15,2 & 10 \\
\hline
\end{tabular}

a: $p^{*}<0,001{ }^{* *}<0,05$. Comparación entre periodontitis crónica vs grupo control (Prueba ji cuadrado /prueba exacta de Fisher).

b: $p^{*}<0,001^{* *}<0,05$. Comparación entre periodontitis agresiva vs grupo control (Prueba ji cuadrado /prueba exacta de Fisher). 
significativas entre los dos grupos con periodontitis.

A. actinomycetemcomitans se presentó con $14,3 \%$ en periodontitis crónica y su frecuencia se elevó ligeramente en periodontitis agresiva a $22,0 \%$; sin embargo, sólo se observaron diferencias significativas entre el grupo de periodontitis agresiva y el grupo control $(p<0,05)$. $E$. corrodens se encontró con mayor frecuencia en los dos grupos con periodontitis $(p<0,001$ con respecto al grupo control). La frecuencia de este microorganismo no mostró diferencias significativas entre los pacientes con periodontitis crónica y periodontitis agresiva, ni entre sus formas localizada y generalizada (datos no mostrados).

La presencia de bacilos entéricos, principalmente los pertenecientes a la tribu Klebsielleae (géneros Klebsiella, Enterobacter, Serratia) fue evidente en todos los grupos analizados. Estas bacterias se presentaron en mayor proporción en periodontitis agresiva $(p<0,05$ comparado con el grupo control) y no existió diferencia significativa entre los dos grupos con periodontitis (cuadro 3).

No se encontró una diferencia significativa entre los géneros (masculino y femenino) de la población analizada y los diferentes microorganismos estudiados (datos no mostrados).

En relación con el nivel socioeconómico de los grupos investigados, $P$. gingivalis y $T$. forsythia se presentaron más frecuentemente en los estratos bajos $(p<0,05)$ y Fusobacterium spp se encontró con mayor frecuencia en los estratos medio y bajo $(p<0,05)$. La colonización por bacilos entéricos fue mayor en los pacientes de estrato bajo, aunque sin diferencia significativa $(p>0,05)$ (cuadro 4).

El recuento de unidades formadoras de colonias por mililitro de cada una de las bacterias (expresado en porcentaje) en relación con el total de la flora cultivable se presenta en la figura 2. $P$. gingivalis se presentó en cantidad elevada en los pacientes con periodontitis crónica $(p<0,05$ comparado con el grupo control), pero no se observaron diferencias significativas entre los grupos con periodontitis. T. forsythia se encontró en elevada concentración en los grupos con periodontitis crónica y periodontitis agresiva $(p<0,05$ en comparación con el grupo control), pero no se observaron diferencias entre los dos grupos con periodontitis. E. corrodens y $P$. intermedia se presentaron en proporción elevada en el grupo con periodontitis agresiva $(p<0,05$ comparado con el grupo control). El recuento de $A$. actinomycetemcomitans y de bacilos entéricos no mostró diferencias significativas entre los grupos estudiados ( $p>0,05$ en comparación con el grupo control).

\section{Discusión}

En este estudio $P$. gingivalis y $T$. forsythia se presentaron con alta prevalencia en periodontitis

Cuadro 3. Frecuencia (\%) de detección de bacilos entéricos (familias Enterobacteriaceae y Pseudomonadaceae) según presentación clínica.

\begin{tabular}{lccr}
\hline Microorganismo & $\begin{array}{c}\text { Periodontitis crónica } \\
(\mathbf{n = 1 2})\end{array}$ & $\begin{array}{c}\text { Periodontitis agresiva } \\
(\mathbf{n = 8})\end{array}$ & $\begin{array}{c}\text { Grupo control } \\
(\mathbf{n}=\mathbf{4})\end{array}$ \\
\hline Cantidad insuficiente para diagnóstico & 12,5 & 0,0 & 0,0 \\
Citrobacter freundii & 12,5 & 8,3 & 0,0 \\
Enterobacter cancerogenenus & 0,0 & 8,3 & 0,0 \\
Enterobacter gergoviae & 0,0 & 8,3 & 0,0 \\
Enterobacter asburiae & 0,0 & 0,0 & 25,0 \\
Enterobacter cloacae & 25,0 & 25,0 & 0,0 \\
Enterobacter sakasakii & 25,0 & 0,0 & 0,0 \\
Klebsiella oxytoca & 0,0 & 8,3 & 50,0 \\
Klebsiella pneumoniae & 0,0 & 16,7 & 0,0 \\
Pseudomonas fluorescens & 12,5 & 0,0 & 0,0 \\
Pantoea agglomerans & 0,0 & 8,3 & 25,0 \\
Serratia licuefaciens & 0,0 & 16,7 & 0,0 \\
Serratia marcescens & 12,5 & 0,0 & 0,0 \\
\hline
\end{tabular}


crónica y periodontitis agresiva, lo que concuerda con resultados obtenidos en Norte América, Japón, Brasil, Chile y México $(14,15,17,25,32-$ $37,43)$. Zambon (44) sugirió que estos dos microorganismos estaban, además, fuertemente asociados con reabsorción ósea, bolsas profundas ( $>5 \mathrm{~mm}$ ), pérdida de adherencia y sangrado al

Cuadro 4. Distribución de frecuencias (\%) de microorganismos periodontopatógenos de acuerdo con el nivel socioeconómico (NSE).

\begin{tabular}{lccr}
\hline Microorganismo & $\begin{array}{r}\text { NSE 1 } \\
(\mathbf{n = 4 6 )}\end{array}$ & $\begin{array}{c}\text { NSE 2 } \\
(\mathbf{n = 8 7})\end{array}$ & $\begin{array}{r}\text { NSE 3 } \\
(\mathbf{n = 5 0 )}\end{array}$ \\
\hline P. gingivalis & $60,9^{*}$ & 49,4 & 32,6 \\
T. forsythia & $63,0^{*}$ & $62,0^{*}$ & 48,9 \\
A. actinomycetemcomitans & 17,4 & 16,1 & 8,2 \\
P. intermedia/nigrescens & 73,9 & 66,7 & 63,2 \\
E. corrodens & 67,4 & 58,6 & 51,0 \\
C. rectus & 63,0 & 54,0 & 36,7 \\
M. micros & 45,7 & 43,7 & 34,7 \\
Fusobacterium spp & $63,0^{*}$ & $65,5^{*}$ & 53,0 \\
D. pneumosintes & 13,0 & 24,1 & 8,2 \\
Bacilos entéricos & 19,6 & 9,2 & 8,2 \\
\hline
\end{tabular}

NSE 1: bajo. 2: medio. 3: alto. ${ }^{*} p<0,05$ (Prueba ji cuadrado/prueba exacta de Fisher). sondaje, lo que coincide con lo reportado por Socransky (11) y con los resultados de este estudio. También se reportó prevalencia importante de $P$. gingivalis en Argentina (38) y de $T$. forsythia en Brasil (34) en pacientes con periodontitis crónica. En este estudio, $P$. gingivalis y $T$. forsythia se encontraron en bajas proporciones en el grupo control, confirmando lo sugerido por Loesche (45), quien observó que $P$. gingivalis puede no encontrarse o estar presente en escasa proporción en los sujetos sanos.

Estudios realizados en diferentes latitudes han reportado que $A$. actinomycetemcomitans es el más frecuente (en altas concentraciones) en periodontitis agresiva, especialmente en Rumania (6), Turquía (27), Tailandia (46), Corea (47) y Holanda (48). Resultados similares se encontraron en Norte América, aunque con menor frecuencia (15). En Grecia (49), por el contrario, se observó que $P$. gingivalis era la bacteria más frecuentemente aislada de pacientes con periodontitis agresiva y $A$. actinomycetemcomitans demostró una prevalencia relativamente baja. En
Sujetos sanos

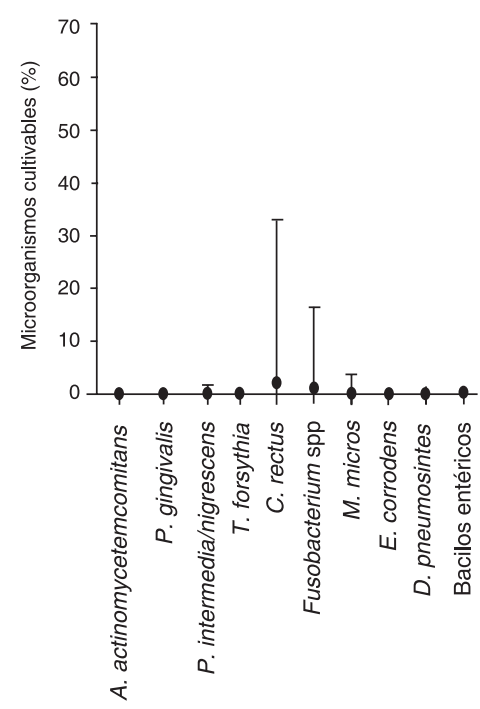

Periodontitis agresiva

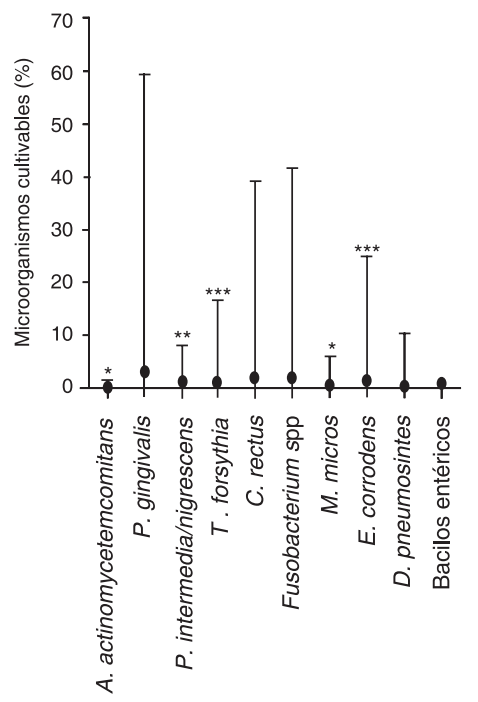

Periodontitis crónica

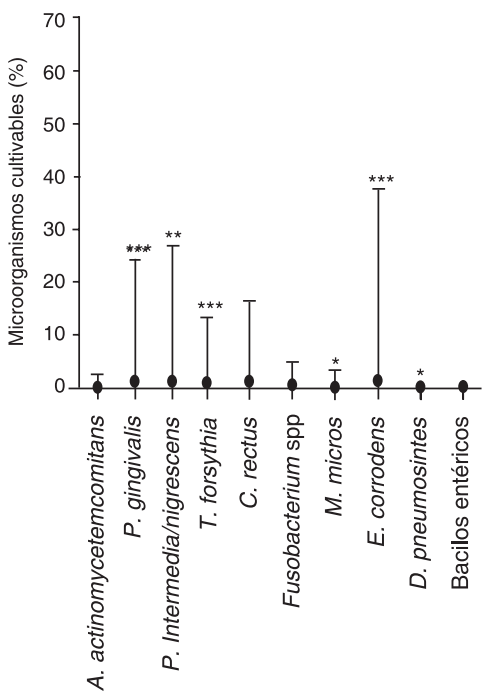

Figura 2. Recuento en porcentaje de microorganismos periodontopatógenos. ${ }^{* * *} p<0,001{ }^{* *} p<0,01{ }^{*} p<0,05$ (Prueba de Mann - Whitney) al comparar periodontitis crónica vs. grupo control y periodontitis agresiva vs. grupo control. 
nuestro estudio el resultado fue similar, ya que se encontró mayor frecuencia de $P$. gingivalis que de $A$. actinomy-cetemcomitans en periodontitis agresiva, lo que coincide con lo reportado en China (3), Japón (25), Kenia (30) y Reino Unido (50). En Sur América, Cortelli (33) en Brasil y Gajardo (35) y López (37) en Chile reportaron que $P$. gingivalis era el microorganismo más frecuente tanto en periodontitis crónica como en periodontitis agresiva. López en Chile (37) encontró alta prevalencia y altos niveles de $P$. gingivalis y $P$. intermedia en los pacientes con periodontitis agresiva, por lo que sugirió que hay poblaciones afectadas por periodontitis agresiva en las cuales existe mayor asociación con estas especies que con $A$. actinomycetemcomitans.

$P$. intermedia/P. nigrescens se encontraron relativamente en las mismas proporciones en los dos grupos con periodontitis $y$, aunque se presentaron diferencias significativas entre ellos y el grupo control, se observó que en los sujetos sanos también se presentaron con una frecuencia elevada. Similares resultados encontraron Nonemacher (20), Van Steenbergen (51) y Conrads (52). Este último reportó $P$. intermedia como la bacteria más frecuente en periodontitis agresiva y sugirió, al igual que Kuboniwa (53), que $P$. nigrescens puede ser un marcador de salud en sujetos sanos. En nuestro estudio no se pudieron diferenciar estas dos especies debido a que las características fenotípicas de las colonias son muy similares en el cultivo.

En este estudio, $C$. rectus se presentó con frecuencia elevada en todos los grupos estudiados, lo que coincide con los hallazgos reportados por Macuch (16) y Rams (54). La alta frecuencia de este microorganismo en los sujetos sanos podría indicarnos que en la población analizada dicha bacteria puede formar parte de la flora normal cuando no está asociada en la biopelícula con otro microorganismo de mayor patogenicidad.

En relación con $M$. micros, aunque Haffajee y Soncransky (55) lo asociaron más con periodontitis agresiva, las proporciones encontradas en nuestro estudio fueron muy similares en los dos grupos enfermos, encontrándose diferencia significativa entre el grupo de periodontitis crónica y el grupo control.

E. corrodens se presentó con frecuencia elevada en los dos grupos con periodontitis y con frecuencia moderada en el grupo control. Chen (19) propuso que E. corrodens puede ser más prevalente en sujetos enfermos que en sanos y Kamma (49), al encontrarla en todas las lesiones periodontales, sugirió que este microorganismo puede representar un papel en la iniciación de la enfermedad; sin embargo, Mayanagy (26) observó que $C$. rectus y $E$. corrodens no estaban relacionados con pérdida de inserción periodontal y que se encontraban frecuentemente en niños sanos, sugiriendo que son comensales de la cavidad oral.

$D$. pneumosintes se presentó en mayor proporción en los dos grupos enfermos que en el grupo control, lo cual coincide con las investigaciones realizadas en pacientes con periodontitis por Contreras (56) y Kamma (57), quienes lo encontraron con mayor frecuencia en sitios activos y observaron coinfección con $P$. gingivalis en $50 \%$ de los sitios activos y en $0 \%$ de los sitios estables, por lo cual propusieron incluirlo entre los patógenos putativos.

En este estudio, Klebsiella, Enterobacter, Serratia (tribu Klebsielleae) fueron los bacilos entéricos más frecuentemente aislados, lo que coincide con estudios relacionados realizados anteriormente $(18,31,32)$. La frecuencia encontrada osciló entre 10 y $15,2 \%$ en los tres grupos de estudio. Investigaciones anteriores han revelado una prevalencia en un rango que oscila entre 14 y $95 \%$ en diferentes poblaciones de Sudan (12), Estados Unidos $(17,18)$, China (24) y varios países de Europa (6), Centro América (31) y Sur América (32). Slots reportó $23 \%$ en California, $14 \%$ en Pennsylvania (18) y $67 \%$ en R. Dominicana (31). En Brasil, Barbosa (32) encontró 31,2\%. Varios investigadores sugieren que la contaminación con estos microorganismos podría deberse a factores como mala higiene oral, ingesta de alimentos contaminados, uso indiscriminado de antibióticos, tiempo prolongado de la muestra en el medio de transporte, autoinoculación con cepillos dentales, raza y ubicación geográfica entre otros. 
Si bien es cierto que en la actualidad el empleo de técnicas moleculares, en especial la reacción en cadena de la polimerasa (PCR), es la metodología más empleada en el diagnóstico de los microorganismos periodontopatógenos por su alta sensibilidad y especificidad, no se puede ignorar la importancia del cultivo, el cual, aunque con menor sensibilidad y especificidad, es considerado el estándar de oro por algunos investigadores y constituye la metodología convencional en el estudio de estas patologías, pues permite la detección simultánea de los microorganismos cultivables presentes en la muestra y la realización de pruebas de sensibilidad antibiótica $(40,58)$. Al comparar estas dos metodologías para el diagnóstico de $A$. actinomycetemcomitans, Ashimoto y col. observaron $71 \%$ de concordancia en los resultados obtenidos (14) y Lau y col. reportaron $18,8 \%$ con PCR en tiempo real y $6,3 \%$ con cultivo (58).

Con $P$. gingivalis se pudieron equiparar los resultados obtenidos con los dos métodos: Lau y col. observaron $81,3 \%$ con PCR en tiempo real y $84,4 \%$ con cultivo (58) y Boutaga y col encontraron $43 \%$ con cultivo y $53 \%$ con PCR en tiempo real (59).

T. forsythia, por el contrario, presentó diferencias considerables al comparar las dos técnicas y se observó que la frecuencia de detección era mayor con PCR debido a que es un organismo "fastidioso" de cultivar. Sin embargo, las diferencias en los resultados también pueden atribuirse a la ubicación geográfica de la población en estudio (58).

En consecuencia, la técnica del cultivo continúa siendo una herramienta útil en este tipo de estudios siempre y cuando se realice en condiciones óptimas (selección apropiada del sitio de la muestra, método utilizado para la recolección de la muestra, transporte, atmósfera, sistema de incubación, utilización de medios selectivos, empleo de pruebas bioquímicas adicionales para la determinación de especie e interpretación de los datos obtenidos) (40). El cultivo ha sido utilizado en estudios epidemiológicos por Slots en República Dominicana en 1991 (31), Nonemacher en Alemania en 2001 (20), van Winkelhoff en Holanda en 2002 (23) y Dogan en Turquía en 2003 (27).

Los resultados obtenidos en la población analizada presentan un perfil microbiológico similar al encontrado en Chile y Brasil. Al igual que en esos países, en periodontitis agresiva predominó $P$. gingivalis y $A$. actinomycetemcomitans se observó con escasa frecuencia. En periodontitis crónica y periodontitis agresiva se presentó alta prevalencia de $P$. gingivalis, $T$. forsythia, $P$. intermedia/P. nigrescens, $C$. rectus, Fusobacterium spp y $E$. corrodens. En los sujetos sanos se encontraron Fusobacterium spp, C. rectus y $P$. intermedia/P. nigrescens con frecuencia elevada y $E$. corrodens y $M$. micros con frecuencia moderada. Los bacilos entéricos se encontraron con baja frecuencia en los tres grupos estudiados.

Los estratos altos de la población mostraron menor frecuencia para la mayoría de las especies estudiadas comparada con los estratos medio y bajo.

Estos resultados constituyen un aporte al estudio del perfil microbiológico de la periodontitis en la población colombiana, el cual es fundamental para formular medidas preventivas y terapéuticas acertadas.

\section{Agradecimientos}

A Marisol Téllez y Luis Fernando Gamboa por el análisis estadístico realizado en el presente estudio. A Jaime E. Castellanos por su acertada orientación para la escritura y presentación de este artículo y a Blanca N. Ruiz por la colaboración en el procesamiento de las muestras.

\section{Conflicto de intereses}

Los autores manifiestan que no existe ningún conflicto de intereses.

\section{Financiación}

El presente estudio se desarrolló gracias a la financiación del Instituto Colombiano para el Desarrollo de la Ciencia y la Tecnología "Francisco José de Caldas" Colciencias, proyecto código: 1308-04-13001 contrato No. RC 470-2002 en convenio con la Universidad El Bosque. 


\section{Referencias}

1. American Academy of Periodontology. International Workshop for a classification of periodontal diseases and conditions. Ann Periodontol 1999;4:8-38.

2. Hamlet SM, Cullinan MP, Westerman B, Lindeman M, Bird PS, Palmer J et al. Distribution of Actinobacillus actinomycetemcomitans, Porphy-romonas gingivalis and Prevotella intermedia in an Australian population. J Clin Periodontol 2001;28:1163-71.

3. Mombelli A, Gmür R, Frey J, Meyer J, Zee KY, Tam $\mathrm{JO}$ et al. Actinobacillus actinomycetemcomitans and Porphyromonas gingivalis in young Chinese adults. Oral Microbiol Immunol 1998;13:231-7.

4. Papapanou PN, Baelum V, Luan WM, Madianos PN, Chen X, Fejerskov $O$ et al. Subgingival microbiota in adult Chinese: Prevalence and relation to periodontal disease progression. J Periodontol 1997;68:651-66.

5. Timmerman MF, Van der Weijden GA, Armand S, Abbas F, Winkel EG, Van Winkelhoff AJ et al. Untreated periodontal disease in Indonesian adolescents. Clinical and microbiological baseline data. J Clin Periodontol 1998;25:215-24.

6. Ali RW, Velcescu C, Jivanescu MC, Lofthus B, Skaug N. Prevalence of 6 putative periodontal pathogens in subgingival plaque samples from Romanian adult periodontitis patients. J Clin Periodontol 1996;23:133-9.

7. Albandar JM. Periodontal diseases in North America. Periodontol 2000 2002;29:31-69.

8. Tovar S, Zúñiga E, Franco A, Jacome S, Ruiz J. III Estudio Nacional en Salud Bucal (ENSAB III). Bogotá: Ministerio de Salud y Centro Nacional de Consultoría CNC; 1999.

9. Shigueo F, Raslan SA, Cortelli JR. Microbial characteristics in periodontal health and disease. Taubate, Brasil: Departamento de Odontología, Universidade de Taubate; 2003.

10. Wilson M. Bacterial biofilms and human disease. Sci Prog 2001;84:235-54.

11. Socransky SS, Haffajee AD, Cugini MA, Smith C, Kent RL Jr. Microbial complexes in subgingival plaque. J Clin Periodontol 1998;25:134-44.

12. Ali RW, Bakken V, Nilsen R, Skaug N. Comparative detection frequency of 6 putative periodontal pathogens in Sudanese and Norwegian adult periodontitis patients. J Periodontol 1994;65:1046-52.

13. Sanz M, van Winkelhoff AJ, Herrera D, DellemijnKippuw N, Simon R, Winkel E. Differences in the composition of the subgingival microbiota of two periodontitis population of different geographical origin. A comparison between Spain and The Netherlands. Eur J Oral Sci 2000;108:383-92.
14. Ashimoto A, Chen C, Bakker I, Slots J. Polymerase chain reaction detection of 8 putative periodontal pathogens in subgingival plaque of gingivitis and advanced periodontitis lesions. Oral Microbiol Immunol 1996;11:266-73.

15. Slots J, Bragd L, Wikström M, Dahlén G. The occurrence of Actinobacillus actinomycetemcomitans, Bacteroides gingivalis and Bacteroides intermedius in destructive periodontal disease in adults. J Clin Periodontol 1986;13:570-7.

16. Macuch PJ, Tanner AC. Campylobacter species in health, gingivitis, and periodontitis. J Dent Res 2000;79:785-92.

17. Tanner A, Maiden MF, Macuch PJ, Murray LL, Kent $\mathbf{R L}$ Jr. Microbiota of health, gingivitis and initial periodontitis. J Clin Periodontol 1998;25:85-98.

18. Slots J, Feik D, Rams TE. Prevalence and antimicrobial susceptibility of Enterobacteriaceae, Pseudomonadaceae and Acinetobacter in human periodontitis. Oral Microbiol Immunol 1990;5:149-54.

19. Chen CK, Wilson ME. Eikenella corrodens in human oral and non-oral infections: A review. J Periodontol 1992;63:941-53.

20. Nonnenmacher C, Mutters R, Jacoby LF. Microbiological characteristics of subgingival microbiota in adult periodontitis, localized juvenile periodontitis and rapidly progressive periodontitis subjects. Clin Microbiol Infect 2001;7:213-7.

21. Müller HP, Heinecke A, Fuhrmann A, Eger T, Zöller L. Intraoral distribution of Actinobacillus actinomycetemcomitans in young adults with minimal periodontal disease. J Periodont Res 2001;36:114-23.

22. Müller HP, Heinecke A, Borneff M, Knopf A, Kiencke C, Pohl S. Microbial ecology of Actinobacillus actinomycetemcomitans, Eikenella corrodens and Capnocytophaga spp in adult periodontitis. J Periodont Res 1997;32:530-42.

23. van Winkelhoff AJ, Loos BG, van der Reijden Wa, van der Velden U. Porphyromonas gingivalis, Bacteroides forsythus and other putative periodontal pathogens in subjects with and without periodontal destruction. J Clin Periodontol 2002;29:1023-8.

24. Sedgley CM, Samaranayake LP, Chan JC, Wei SH. A 4-year longitudinal study of the oral prevalence of enteric gram-negative rods and yeasts in Chinese children. Oral Microbiol Immunol 1997;12:183-8.

25. Takeuchi Y, Umeda M, Ishizuka M, Huang $\mathbf{Y}$, Ishikawa I. Prevalence of periodontopathic bacteria in aggressive periodontitis patients in a Japanese population. J Periodontol 2003;74:1460-9.

26. Mayanagy G, Sato T, Shimauchi H, Takahashi N. Detection frequency of periodontitis-associated bacteria by polymerase chain reaction in subgingival 
and supragingival plaque of periodontitis and healthy subjects. Oral Microbiol Immunol 2004;19:379-85.

27. Dogan B, Antinheimo J, Cetiner D, Bodur A, Emingil G, Buduneli $E$ et al. Subgingival microflora in Turkish patients with periodontitis. J Periodontol 2003; 74:803-14.

28. Ali RW, Johannessen AC, Dahlen J, Socransky SS, Skaug N. Comparison of the subgingival microbiota of periodontally healthy and diseased adults in Northern Cameroon. J Clin Periodontol 1997;24:830-5.

29. Ali RW, Skaug N, Nilsen R, Bakken V. Microbial associations of 4 putative periodontal pathogens in Sudanese adult periodontitis patients determined by DNA probes analysis. J Peridontol 1994;65:1053-7

30. Dahlen G, Manji F, Baelum V, Fejerskov O. Black pigmented bacteroides species and A. actinomycetemcomitans in subgingival plaque of adult Kenyans. J Clin Periodontol 1989;16:305-10.

31. Slots J, Rams TE, Feik D, Taveras HD, Gillespie GM. Subgingival microflora of advanced periodontitis in the Dominican Republic. J Periodontol 1991;62:543-7.

32. Barbosa FC, Mayer MP, Saba-Chuifi E, Cai S. Subgingival occurrence and antimicrobial susceptibility of enteric rods and pseudomonads from Brazilian periodontitis patients. Oral Microbiol Immunol 2001;16:306-10.

33. Cortelli JR, Cortelli SC, Jordan S, Haraszthy VI, Zambon JJ. Prevalence of periodontal pathogens in Brazilians with aggressive or chronic periodontitis. J Clin Periodontol 2005;32:860-6.

34. Klein MI, Goncalves RB. Detection of Tannerella forsythensis (Bacteroides forsythus) and Porphyromonas gingivalis by polymerase chain reaction in subjects with different periodontal status. J Periodontol 2003; 74:798-802.

35. Gajardo M, Silva N, Gómez L, León R, Parra B, Contreras A et al. Prevalence of periodontopathic bacteria in aggressive periodontitis patients in a Chilean population. J Periodontol 2005;76:289-94.

36. López NJ. Occurrence of Actinobacillus actinomycetemcomitans, Porphyromonas gingivalis and Prevotella intermedia in progressive adult periodontitis. J Periodontol 2000;71:948-54.

37. López NJ, Mellado JC, Leighton GX. Occurrence of Actinobacillus actinomycetemcomitans, Porphyromonas gingivalis, and Prevotella intermedia in juvenile periodontitis. J Clin Periodontol 1996;23:101-5.

38. Nogueira MA, Fernandez CL, Furman C, Chiappe $\mathbf{V}$, Marcantoni $\mathbf{M}$, Bianchini $\mathbf{H}$. Clinical and microbiological study of adult periodontal disease. Rev Argent Microbiol 2001;33:133-40.

39. Departamento Administrativo de Planeación Distrital. Estratificación. [Consultada: mayo 5 de 2006].
Disponible en: http://www.dapd.gov.co/www/section1950.jsp

40. Slots J. Rapid identification of important periodontal microorganisms by cultivation. Oral Microbiol Immunol 1986;1:48-57.

41. Chen C, Slots J. Microbiological tests for Actinobacillus actinomycetemcomitans and Porphyromonas gingivalis. Periodontol 2000 1999;20:53-64.

42. Slots J. Detection of colonies of Bacteroides gingivalis by a rapid fluorescence assay for trypsin-like activity. Oral Microbiol Immunol 1987;2:139-41.

43. Almaguer-Flores A, Jacobo-Soto V, SánchezVargas LO, Lara-Córdoba M, Alcántara-Maruri E, Ximénez-Fyvie LA. Descripción de la microbiota subgingival de sujetos mexicanos con periodontitis crónica. Rev Odont Mex 2005;9:7-15.

44. Zambon JJ. Periodontal disease: Microbial factors. Ann Periodontol 1996;1:879-925.

45. Loesche WJ. DNA probe and enzyme analysis in periodontal diagnostics. J Periodontol 1992;63(12 Suppl):1102-9.

46. Dahlen G, Widar F, Teanpaisan R, Papapanou PN, Baelum V, Fejerskov 0 . Actinobacillus actinomycetemcomitans in a rural adult population in Southern Thailand. Oral Microbiol Immunol 2002; 17:137-42.

47. Choi BK, Park SH, Yoo JY, Choi SH, Chai JK, Cho $\mathrm{KS}$ et al. Detection of major putative periodontopathogens in Korean advanced adult periodontitis patients using a nucleic acid based approach. J Periodontol 2000;71:1387-94.

48. Van der Weijden GA, Timmerman MF, Reijerse E, Wolffe GN, van Winkelhoff AJ, Van der Velden U. The prevalence of $A$. actinomycetemcomitans, $P$ gingivalis, and $P$. intermedia in selected subjects with periodontitis. J Clin Periodontol 1994;21:583-8.

49. Kamma JJ, Nakou M, Gmür R, BaehnI PC. Microbiological profile of early onset/aggressive periodontitis patients. Oral Microbiol Immunol 2004;19:314-21

50. Mullally BH, Dace B, Shelburne CE, Wolff LF, Coulter WA. Prevalence of periodontal pathogens in localized and generalized forms of early-onset periodontitis. J Periodontal Res 2000;35:232-41.

51. van Steenbergen Tj, Van der Velden U, Abbas F, de Graaff J. Microflora and bacterial DNA restriction enzyme analysis in young adults with periodontitis. J Periodontol 1991;62:235-41.

52. Conrads G, Mutters R, Fischer J, Brauner A, Lutticken R, Lampert F. PCR reaction and dot-blot hybridization to monitor the distribution of oral pathogens within plaque samples of periodontally healthy individuals. J Periodontol 1996;67:994-1003. 
53. Kuboniwa M, Amano A, Kimura KR, Sekine S, Kato S, Yamamoto Y et al. Quantitative detection of periodontal pathogens using real-time polymerase chain reaction with TaqMan probes. Oral Microbiol Immunol 2004;19:168-76.

54. Rams TE, Feik D, Slots J. Campylobacter rectus in human periodontitis. Oral Microbiol Immunol 1993;8;4:230-5

55 Haffajee AD, Socransky SS. Microbial etiological agents of destructive periodontal diseases. Periodontol 2000 1994;5:78-111.

56. Contreras A, Doan N, Chen C, Rusitanonta T, Flynn MJ, Slots J. Importance of Dialister pneumosintes in human periodontitis. Oral Microbiol Immunol 2000;15:269-72.
57. Kamma JJ, Contreras A, Slots J. Herpes viruses and periodontopathic bacteria in early-onset periodontitis. J Clin Periodontol 2001;28:879-85.

58. Lau L, Sanz M, Herrera D, Morillo JM, Martín C, Silva A. Quantitative real-time polymerase chain reaction versus culture: a comparison between two methods for the detection and quantification of Actinobacillus actinomycetemcomitans, Porphyromonas gingivalis and Tannerella forsythensis in subgingival plaque samples. J Clin Periodontol 2004;31:1061-9.

59. Boutaga K, van Winkelhoff AJ, VandenbrouckeGrauls CM, Savelkoul PH. Comparison of real-time PCR and culture for detection of Porphyromonas gingivalis in subgingival plaque samples. J Clin Microbiol 2003;41:4950-4 